\title{
Oncogene MYCN regulates localization of NKT cells to the site of disease in neuroblastoma
}

\author{
Liping Song, ${ }^{1}$ Tasnim Ara, ${ }^{1}$ Hong-Wei Wu, ${ }^{1}$ Chan-Wook Woo, ${ }^{2}$ C. Patrick Reynolds, ${ }^{1}$ \\ Robert C. Seeger, ${ }^{1,3}$ Yves A. DeClerck, ${ }^{1}$ Carol J. Thiele, ${ }^{2}$ \\ Richard Sposto, ${ }^{1,4}$ and Leonid S. Metelitsa ${ }^{1}$
}

\begin{abstract}
'Division of Hematology-Oncology, Department of Pediatrics, Childrens Hospital Los Angeles (CHLA) and Keck School of Medicine, University of Southern California, Los Angeles, California, USA. ${ }^{2}$ Pediatric Oncology Branch, Center for Cancer Research, National Cancer Institute, National Institutes of Health, Bethesda, Maryland, USA. ${ }^{3}$ Children's Oncology Group, Arcadia, California, USA. ${ }^{4}$ Department of Preventive Medicine, Keck School of Medicine, University of Southern California, Los Angeles, California, USA.
\end{abstract}

\begin{abstract}
Vo24-invariant natural killer T (NKT) cells are potentially important for antitumor immunity. We and others have previously demonstrated positive associations between NKT cell presence in primary tumors and long-term survival in distinct human cancers. However, the mechanism by which aggressive tumors avoid infiltration with NKT and other T cells remains poorly understood. Here, we report that the v-myc myelocytomatosis viral related oncogene, neuroblastoma derived (MYCN), the hallmark of aggressive neuroblastoma, repressed expression of monocyte chemoattractant protein-1/CC chemokine ligand 2 (MCP-1/CCL2), a chemokine required for NKT cell chemoattraction. MYCN knockdown in $M Y C N$-amplified neuroblastoma cell lines restored CCL2 production and NKT cell chemoattraction. Unlike other oncogenes, MYCN repressed chemokine expression in a STAT3-independent manner, requiring an E-box element in the CCL2 promoter to mediate transcriptional repression. MYCN overexpression in neuroblastoma xenografts in NOD/SCID mice severely inhibited their ability to attract human NKT cells, T cells, and monocytes. Patients with $M Y C N$-amplified neuroblastoma metastatic to bone marrow had 4-fold fewer NKT cells in their bone marrow than did their nonamplified counterparts, indicating that the MYCN-mediated immune escape mechanism, which we believe to be novel, is operative in metastatic cancer and should be considered in tumor immunobiology and for the development of new therapeutic strategies.
\end{abstract}

\section{Introduction}

Va24-invariant natural killer T (NKT) cells comprise an evolutionarily conserved sublineage of $\mathrm{T}$ cells that is characterized by reactivity to self- and microbial-derived glycolipids presented by the monomorphic HLA class I-like molecule CD1d. NKT cells may serve as an early source of cytokines that provide initial signals for other cells of the immune system to initiate innate and adaptive responses $(1,2)$. The potential of NKT cells has been demonstrated in numerous models of cancer $(3,4)$. Selective decreases of NKT cell number and/or functional activity have been reported in patients with diverse types of cancer (5-7), and a recent study demonstrated that low levels of circulating NKT cells predict a poor clinical outcome in patients with head and neck squamous cell carcinoma (8). We previously demonstrated that NKT cells infiltrated primary tumors in a subset of patients with stage 4 neuroblastoma and that this was associated with 2-fold longer diseasefree survival (9). Another recent study demonstrated that NKT cell infiltration in primary tumors served as an independent factor of favorable outcome in patients with colorectal cancers (10). These

Nonstandard abbreviations used: CBA, cytometric bead array; CCL, CC chemokine ligand; CXCL, CXC chemokine ligand; EGFP, enhanced GFP; FACS, fluorescence-activated cell sorting; $\alpha$ GalCer, $\alpha$-galactosylceramide; INR, initiator; MCP-1, monocyte chemoattractant protein-1; MYCN, v-myc myelocytomatosis viral related oncogene, neuroblastoma derived; MYCNkd, MYCN knockdown; NKT, V $\alpha 24$-invariant natural killer T (cell); PBL, peripheral blood lymphocyte; shRNA, short hairpin RNA.

Conflict of interest: The authors have declared that no conflict of interest exists.

Citation for this article: J. Clin. Invest. 117:2702-2712 (2007). doi:10.1172/JCI30751. findings suggest that NKT cell localization to the tumor site may be important for the initiation of effective antitumor immune responses. Hence, tumors may acquire strategies to avoid NKT cells via as-yet-unknown mechanisms.

Accumulating evidence suggests that oncogenic signaling may not only provide a cell-intrinsic advantage for tumor cells, but also mediate immune evasion (11). In particular, STAT3 is frequently activated in diverse types of cancer, causes tumor growth, and mediates profound immune suppression by inhibiting the expression of chemokines required for immune effector cell migration to the tumor site and by inducing immunosuppressive factors, such as IL-10 and VEGF, that inhibit DC differentiation and maturation (12).

In a recent report, we demonstrated that NKT cells failed to localize to primary neuroblastoma tumors that expressed high levels of the v-myc myelocytomatosis viral related oncogene, neuroblastoma derived $(M Y C N)$ protooncogene (9). We also observed an inverse correlation between the RNA levels of $M Y C N$ and monocyte chemoattractant protein-1/CC chemokine ligand 2 (MCP-1/ CCL2). CCL2 is a prototypic inflammatory chemokine, which was initially described as a major chemoattractant for monocytes (13, 14) and later demonstrated to be one of the most potent chemokines for both CD4 and CD8 effector-memory $\alpha / \beta$ T cells, $\gamma / \delta$ T cells $(15,16)$, and NKT cells $(17,18)$ that express its receptor, CC chemokine receptor 2 (CCR2). We showed that CCL2 was required for NKT cell chemoattraction by neuroblastoma cells in vitro (9).

These data suggested that MYCN might be involved in the mechanism of immune escape in neuroblastoma. MYC family tran- 
A
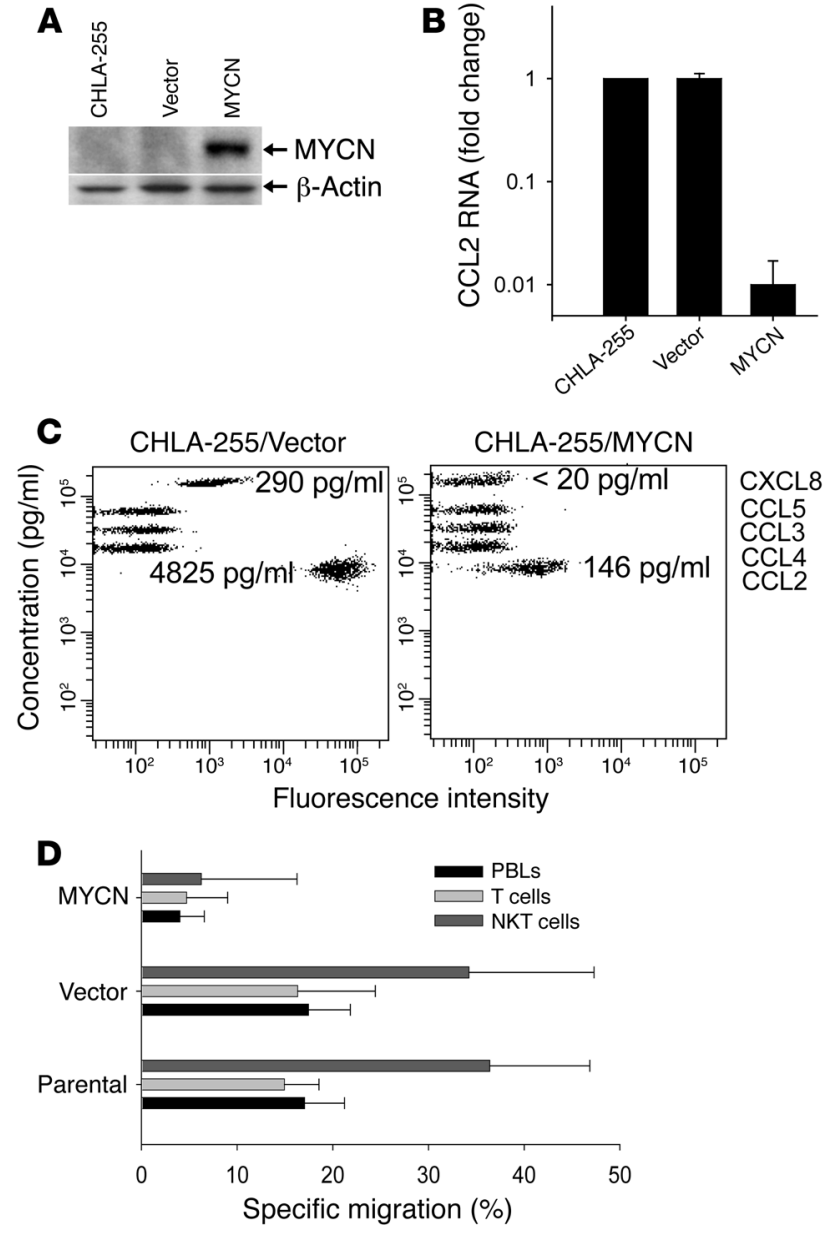

scription factors are highly conserved evolutionarily from yeast to mammals (19). Three members of the family (MYCC, MYCN, and $M Y C L$ ) are protooncogenes; their expression has been linked to a wide variety of cancers $(20,21) . M Y C N$ is overexpressed as a result of genomic amplification in $25 \%$ of patients with neuroblastoma, and these patients have unfavorable prognoses regardless of other parameters (22). MYCN activates transcription of genes involved in cell-cycle progression (23) and represses genes that induce cell differentiation (24). Little is known, however, about MYCN involvement in the regulation of immunologically relevant genes. In this study, we investigated the cause-effect relationship among MYCN, CCL2, and NKT cell localization to the tumor site. We demonstrate that MYCN, via repression of CCL2 expression in neuroblastoma cells, prevented NKT cell localization to the tumor site in an in vivo model of neuroblastoma and in patients with metastatic disease.

\section{Results}

MYCN overexpression abrogates CCL2 expression in neuroblastoma cells and NKT cell chemoattraction. To test whether MYCN negatively regulates MCP-1/CCL2 expression in neuroblastoma cells, we transduced MYCN-low, CCL2-high neuroblastoma cell line CHLA-255 with MYCN cDNA using a lentiviral expression vector. After selection and expansion, cells stably expressed high levels of MYCN that were comparable to those in $M Y C N$-amplified primary neuroblastoma cells or cell lines (Figure 1A and Figure 2A). MYCN overexpression

\section{Figure 1}

MYCN represses CCL2 expression and inhibits the chemoattraction of NKT and T cells. CHLA-255 neuroblastoma cells were stably transduced with MYCN cDNA or empty vector control. (A) Representative Western blot analysis of MYCN protein in the nuclear extracts from indicated cells. (B) CCL2 RNA expression was quantified by TaqMan RT-PCR; values are relative to expression in parental cells. Results are mean \pm SD from 3 experiments. (C) CBA analysis. Five indicated chemokines were measured in supernatants collected after 24 hours' culture of $80 \%$ confluent cells. Chemokine concentration is proportional to the fluorescence intensity on the $x$ axis and was calculated by standard curve analysis. Concentrations above $20 \mathrm{pg} / \mathrm{ml}$ are shown. (D) The same supernatants as in $\mathbf{C}$ were placed in lower chambers of dual-chamber plates with 5 - $\mu \mathrm{m}$ pore membrane. Freshly isolated human monocyte-depleted PBLs were added in upper chambers and allowed to migrate for 4 hours. Absolute numbers of NKT and $T$ cells were determined by flow cytometry using CD3/6B11 staining and TruCOUNT tubes with a known number of beads (see Methods). Percent migration reflects the proportion of migrated cells relative to input cells. Specific migration was calculated by subtracting the number of cells that migrated to control medium. Results are mean \pm SD from 4 experiments.

in CHLA-255 cells (CHLA-255/MYCN) resulted in a more than 100 -fold decrease of CCL2 RNA expression, as assessed by quantitative RT-PCR, compared with that in vector control or parental cells (Figure 1B). Multiplexing cytometric bead array (CBA) analysis revealed that MYCN overexpression led to a 33-fold decrease of CCL2 protein secretion in CHLA-255 cells (Figure 1C). In addition, modest expression of CXC chemokine ligand 8/IL-8 (CXCL8/IL-8) in these cells was abrogated after MYCN overexpression, while the levels of 3 other measured chemokines (CCL5/RANTES, CCL3/ MIP1 $\alpha$, and CCL4/MIP1 $\beta$ ) remained undetectable regardless of MYCN status. To examine whether MYCN overexpression in neuroblastoma cells affects their chemoattractive potential, we performed in vitro migration assay with freshly isolated peripheral blood lymphocytes (PBLs). We counted the absolute number of migrating $\mathrm{T}$ and NKT cells by fluorescence-activated cell sorting (FACS) using TruCOUNT tubes with a known number of preloaded beads as we previously described $(9,25)$. We also quantified migration of monocytes (defined by scatter parameters and CD14 expression) from PBMCs in separate experiments, because these cells were the first to migrate to CCL2 and might influence subsequent migration of other leukocytes (Supplemental Figure 1A). The supernatants from CHLA-255/MYCN cells had minimal chemoattractive activity for NKT cells, T cells, or monocytes, whereas on average $35 \%$ of NKT cells, $15 \%$ of T lymphocytes, and $46 \%$ of monocytes migrated toward supernatants from the parental or vector control neuroblastoma cells $(P<0.001$; Figure 1D). Therefore, MYCN repressed CCL2 expression in neuroblastoma cells that rendered them nearly incapable of chemoattracting NKT cells, $\mathrm{T}$ cells, and monocytes. The reduction of NKT cell number in $M Y C N$-amplified tumors could also be explained if MYCN overexpression rendered neuroblastoma cells cytotoxic against infiltrating NKT cells. To test this possibility, we cocultured CHLA-255 and CHLA-255/MYCN neuroblastoma cells with PBMCs (up to a 1:1 ratio) and examined induction of apoptosis in T and NKT cells by FACS using annexin $\mathrm{V}$ staining in combination with anti-CD3 and anti-V $\alpha 24-\mathrm{J} \alpha 18$ (6B11) mAbs, respectively, as we previously described (25). No toxicity was observed either against primary $\mathrm{T}$ or NKT cell in 24 hours of culture (data not shown). 

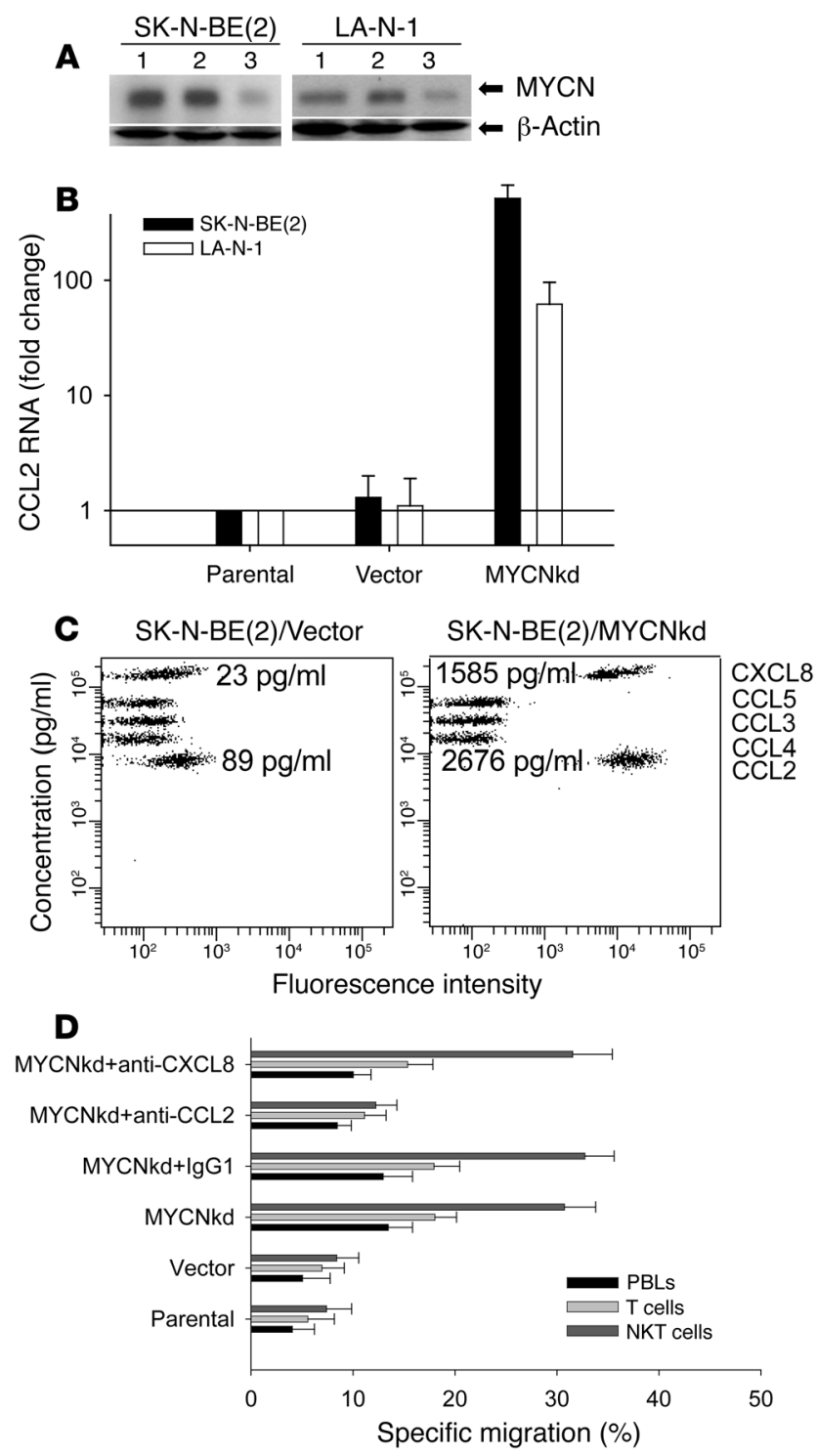

MYCN knockdown induces CCL2 expression in neuroblastoma cells and NKT cell chemoattraction. To examine whether MYCN is required for the control of neuroblastoma cell potential to chemoattract NKT cells, we used an enhanced GFP-containing (EGFP-containing), lentiviral vector-delivered short hairpin RNA (shRNA) to knock down MYCN expression in SK-N-BE(2) and LA-N-1 MYCNamplified cell lines. EGFP-positive cells were sorted by FACS; after expansion, these cells demonstrated 80\%-95\% MYCN downregulation by Western blot analysis (Figure 2A). After MYCN knockdown (MYCNkd), cells grew at a 4- to 8-fold slower rate and, unlike their parental or vector control counterparts, failed to establish tumor xenografts in NOD/SCID mice (data not shown). Quantitative RT-PCR analysis revealed a 65- to 930-fold increase of CCL2 RNA expression in MYCNkd cells compared with that of parental or vector control cells (Figure 2B). CBA analysis showed a 30-fold upregulation of CCL2 secretion in SK-N-BE(2)/MYCNkd cells (Figure 2C). CXCL8 production was also induced after MYCNkd, whereas the levels of 3 other tested chemokines (CCL5, CCL3, and CCL4) remained undetectable. Despite the increase in CCL2 RNA

\section{Figure 2}

MYCNkd induces CCL2 expression in neuroblastoma cells and NKT cell chemoattraction. SK-N-BE(2) and LA-N-1 MYCN-amplified neuroblastoma cell lines were stably transduced with MYCN shRNA using a U6 expression cassette into pHIV-7-EGFP lentiviral plasmid. (A) Representative Western blot analysis of MYCN protein in the nuclear extracts from indicated cells. Lane 1, parental; lane 2, vector control; lane 3, MYCN shRNA. (B) CCL2 RNA expression was quantified by TaqMan RT-PCR; values are relative to expression in parental cells. Results are mean \pm SD from 3 experiments. (C) CBA analysis was performed with supernatants of vector control or MYCN shRNA-transduced SK-N-BE(2) cells as described in Figure 1C. (D) Migration of freshly isolated PBLs to the neuroblastoma cell supernatants with the indicated conditions was performed as described in Figure 1C. Where indicated, supernatants were pretreated for 1 hour with neutralizing anti-CCL2, anti-CXCL8, or isotype control (IgG1) mAb. Results are mean \pm SD from 3 experiments.

expression in both cell lines, LA-N-1/MYCNkd cells failed to produce CCL2 protein (data not shown). In the analysis of PBL in vitro chemoattraction, we found that NKT cell migration to the supernatant from SK-N-BE(2)/MYCNkd cells increased 5-fold compared with vector control cells $(P<0.001)$; this effect was reversed by anti-CCL $2 \mathrm{mAb}$ but not by anti-CXCL8 neutralizing $\mathrm{mAb}$ (Figure 2D). The migration of other T cells (Figure 2D) and monocytes (Supplemental Figure 1B) also increased from mean values of $7 \%$ and $10 \%$ to $18 \%$ and $39 \%$, respectively $(P<0.001)$. Therefore, in $M Y C N$-amplified neuroblastoma cells, MYCN controls their ability to chemoattract NKT cells, T cells, and monocytes.

$M Y C N$ does not affect STAT3 and represses CCL2 via direct binding to its promoter. Two recent reports demonstrated that constitutive activation of the STAT3 signaling pathway couples oncogenic transformation with immune escape via repression of chemokine genes in tumor cells and, via soluble mediators, inhibition of DC activation or maturation $(26,27)$. Therefore, we tested whether MYCN affects STAT3 expression and activation in neuroblastoma cells. Western blot analysis showed that MYCN overexpression in CHLA-255 cells affected neither the level of STAT3 protein expression nor its phosphorylation status (Figure 3A), whereas treatment with IL-6 induced rapid STAT3 phosphorylation, demonstrating that this pathway is intact in neuroblastoma cells. Furthermore, supernatants from either parental or MYCN-transduced neuroblastoma cells had no or little stimulatory effect on human DCs (increased mean CD86 expression in $\mathrm{CD}^{2} 6^{+}$cells) derived from peripheral blood monocytes, whereas supernatant from IL-6-treated parental cells decreased DC size (forward scatter) by nearly $20 \%(P<0.01$; data not shown) and significantly downregulated CD86 expression on the DC surface compared with supernatants from untreated CHLA-255 cells ( $P<0.001$; Figure 3B).

Although in most cases MYC members act as transcriptional activators, they can also mediate repression of some genes via binding to E-box or initiator-like (INR-like) elements $(28,29)$. Using the GenBank database (http://www.ncbi.nlm.nih.gov/Genbank/), we analyzed the CCL2 promoter/enhancer region and detected 1 E-box (CATGTG) and 3 INR elements (CTCACTTCT, TTCACCTTC, and CCCATCCTCCC) that were located at positions $-2,713,-2,595$, $-2,474$, and -225 , respectively. The mechanism of MYC-mediated repression is often associated with MYC interaction with an activating transcription factor that binds to the same or a nearby site on the promoter (24). To test whether an E-box-containing site of the CCL2 promoter is required for CCL2 expression and/or MYCN- 
A

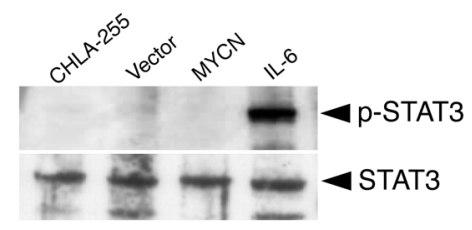

B
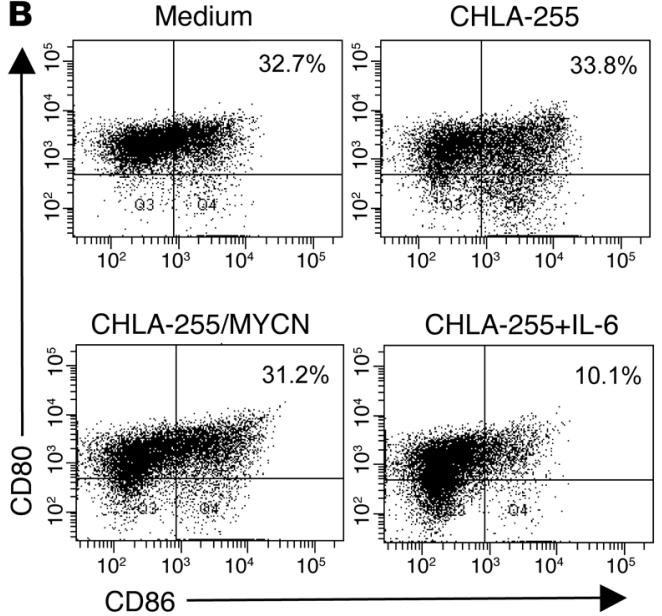

mediated repression in neuroblastoma cells, we cut a 200-bp E-box-containing region from the 2.8-kb CCL2 promoter construct. The remaining $2.6-\mathrm{kb}$ promoter region containing all 3 INR sites almost completely lost activity in CHLA-255 cells in the promoter assay (Figure 4A), indicating that the E-box-containing region is required for CCL2 expression. In CHLA-255/MYCN cells, the promoter activity was lost even with the 2.8 -kb E-box-containing construct, suggesting that MYCN may bind to E-box and interfere with an activating factor. EMSA revealed strong binding of the E-box DNA probe to the nuclear extract from MYCN-overexpressing cells, whereas little binding (consistent with the native single-copy MYCN expression) was observed to the extract of vector control cells (Figure 4B). E-box specificity was confirmed by competition with a nonlabeled E-box probe. Anti-MYCN mAb, but not isotype control $\mathrm{mAb}$, prevented the appearance of the E-boxspecific band, indicating that this DNA-protein complex contains MYCN (Figure 4B). In contrast, specific probes for each of 3 INR sites (Figure 4C) and a proximal SP1 site (data not shown) failed to bind MYCN in the same conditions. Therefore, MYCN binds to an E-box, a consensus MYC family site, in the CCL2 promoter and represses its activity in neuroblastoma cells.

NKT and T cells fail to infiltrate MYCN-overexpressing neuroblastoma xenografts in BM of NOD/SCID mice. Because bone/BM is the most common site for metastases and disease recurrence in neuroblastoma and many other types of cancer, we used a previously described bone/BM model of neuroblastoma (30) to test the in vivo effect of MYCN on NKT cell localization to the tumor site. We inoculated xenografts of parental CHLA-255 cells and CHLA-255/MYCN cells in the BM cavities of left and right femurs of the same NOD/SCID mice (Figure 5A). After 4 weeks, in vitro-expanded human NKT cells were injected i.v. together with PBMCs (1:10 ratio, $5 \times 10^{7}$ total cells). Mice were sacrificed 24 hours after PBMC transfer because our kinetics experiments (data not shown) and a recent report (31) indicated that the majority of human leukocytes, including NKT cells, reach BM in NOD/SCID mice by 24 hours. The numbers of

\section{Figure 3}

MYCN neither activates STAT3 nor affects DC phenotype. (A) Lysates from parental, vector-, or MYCN-transduced CHLA-255 cells were examined for phosphorylated STAT3 (p-STAT3) and for STAT3 by Western blot. Treatment with recombinant human IL-6 $(10 \mathrm{ng} / \mathrm{ml}$ for 15 minutes) was used as a positive control. (B) Flow cytometric analysis of viable DCs generated in one-half volume conditioned medium from indicated neuroblastoma cells. One-half volume Iscove's modified Dulbecco's medium and conditioned medium from IL-6-treated (24 hours) CHLA-255 cells were used as negative and positive controls, respectively. Numbers in plots denote percent CD80+CD86+ cells. Shown are representative plots from 3 experiments.

neuroblastoma cells and human leukocytes, including lymphocyte subsets and monocytes, were examined in BM of both femurs and in tumor-free tibias as a control using multicolor flow cytometry (Figure 5A and Supplemental Figures 2-4). We tested the null hypothesis that the percentage of human leukocytes among mouse BM cells would be unaffected by the presence of neuroblastoma cells and their MYCN status. The results demonstrate that despite a slightly higher frequency of CHLA-255/MYCN cells compared with CHLA-255 cells (difference between means, $5.2 \% \pm 1.7 \%$; $P=0.006$, Student's $t$ test), the rate of leukocyte infiltration was significantly lower in the femur with the CHLA-255/MYCN cells. The presence of CHLA-255 cells in the mouse BM enhanced infiltration with human $\mathrm{T}$ and NKT cells by more than 10 -fold compared with control (mean T cells, $1.52 \%$ versus $0.14 \%$; mean NKT cells, $0.047 \%$ versus $0.0043 \% ; P<0.0001$ for both comparisons, paired Student's $t$ test). In contrast, the presence of CHLA-255/MYCN cells only slightly enhanced $\mathrm{T}$ cell (mean, $0.225 \%$ versus $0.14 \%$; $P=0.043)$ and NKT cell $(0.0087 \%$ versus $0.0043 \% ; P=0.021$, respectively) infiltration. The mean differences in the percentage of $\mathrm{T}$ and NKT cells between CHLA-255 and CHLA-255/MYCN sites were both significant $(P<0.0001)$.

In contrast to input PBMC plus NKT preparation, in which $\mathrm{CD}^{+} \mathrm{T} / \mathrm{NKT}$ cells constituted about $75 \%$ of lymphocytes, BMinfiltrating lymphocytes were almost exclusively $\mathrm{CD} 3^{+}$cells. There was 3-fold enrichment of $\mathrm{CD}^{+}{ }^{+}$cells with CD45RA-CCR7phenotype (Supplemental Figure 2), which is characteristic for effector-memory T cells and all NKT cells $(18,32)$. We did not find significant changes in the distribution of $\mathrm{CD} 4 / \mathrm{CD} 8$ subsets in $\mathrm{T}$ and NKT cells in BM versus input cells (Supplemental Figure 3). The presence of neuroblastoma cells or their MYCN status had no significant effect on the subset distribution, although very low frequencies of human leukocytes in control and CHLA255/MYCN BM decreased the accuracy of subset discrimination (data not shown).

Human monocytes were identified as $\mathrm{CD} 3{ }^{-} \mathrm{CD} 14^{+}$cells after gating on human $\mathrm{CD}^{4} 5^{+}$leukocytes (Supplemental Figure 4). Regardless of the experimental conditions, about $99 \%$ of non-T/NKT leukocytes that infiltrated murine BM were monocytes. As shown in Figure 5B, we observed a 9.6-fold increase in monocyte infiltration in CHLA- 255 versus control BM $(0.134 \%$ versus $0.014 \%$; $P<0.0001)$ and a 1.9 -fold increase in CHLA-255/MYCN versus control BM $(0.027 \%$ versus $0.014 \% ; P=0.052)$. The frequencies of B $\left(\mathrm{CD} 45^{+} \mathrm{CD} 3{ }^{-} \mathrm{CD} 19^{+}\right)$and $\mathrm{NK}\left(\mathrm{CD} 45^{+} \mathrm{CD} 33^{-} \mathrm{CD} 56^{+}\right)$cells were beyond the threshold of FACS resolution (data not shown).

Thus, following adoptive transfer of human PBMCs, effectormemory T cells, NKT cells, and monocytes selectively migrated to the murine $\mathrm{BM}$, and their migration was enhanced by MYCN non- 

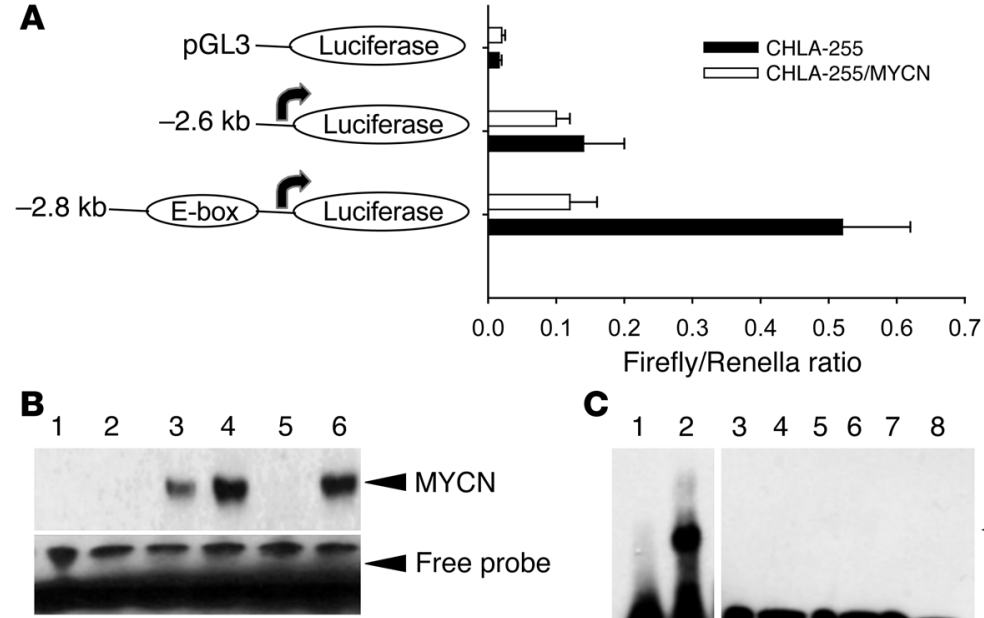

C

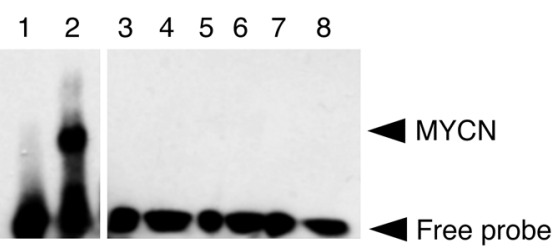

Figure 4

MYCN directly binds CCL2 promoter. (A) CCL2 promoter CDNA constructs with $(2.8 \mathrm{~kb})$ and without (2.6 kb) E-box site were fused with a Firefly luciferase cDNA. A pGL3 plasmid containing only Firefly luciferase cDNA was used as a negative control. CHLA-255 or CHLA-255/MYCN cells were transiently cotransfected with the indicated constructs and pRLSV40 plasmid containing Renilla luciferase cDNA as an internal control. The activity of CCL2 promoter was detected in a dual-luciferase assay and expressed as a ratio of Firefly to Renilla luciferase luminescence intensity. (B) Nuclear extract from neuroblastoma cells was analyzed by EMSA with biotinylated E-box probe from CCL2 promoter. Lane 1, free probe; lane 2, nonbiotin (cold) probe plus probe plus CHLA255/MYCN; lane 3, probe plus CHLA-255/vector; lane 4, probe plus CHLA-255/MYCN; lane 5, probe plus CHLA-255/MYCN plus anti-MYCN mAb; lane 6, probe plus CHLA-255/MYCN plus IgG control. Data are representative of 6 experiments. (C) Nuclear extract from CHLA-255/MYCN was analyzed by EMSA with E-box probe as above (positive control) or with INR probes. Lane 1, free E-box probe; lane 2, E-box probe plus CHLA-255/MYCN; lanes 3, 5, and 7, free probes for INR-1, INR-2, and INR-3, respectively; lanes 4, 6, and 8, CHLA-255/MYCN plus probes for INR-1, INR-2, and INR-3, respectively. Data are representative of 3 experiments.

amplified neuroblastoma xenograft. MYCN overexpression drastically reduced the ability of neuroblastoma cells to attract these leukocyte subsets to the tumor site in a NOD/SCID mouse model.

CCL2 is required for NKT and T cell localization to the neuroblastoma xenografts in BM of NOD/SCID mice. To examine whether leukocyte migration to BM containing CHLA-255 cells depends on CCL2 or CXCL8, we treated mice with anti-human CCL2 or CXCL8 neutralizing antibodies 24 hours before adoptive transfer of human leukocytes. Figure 6 demonstrates that anti-CCL2 mAb inhibited migration of T cells, NKT cells, and monocytes by $69.4 \%, 76.9 \%$, and $87.9 \%$, respectively $(P<0.001)$. Neither IgG control nor anti-CXCL8 antibodies had a significant effect on leukocyte migration. This finding indicates that CCL2 is required for the ability of neuroblastoma cells to attract these leukocyte subsets in vivo and that MYCN-mediated repression of the CCL2 gene explains the failure of leukocyte migration to MYCN-overexpressing neuroblastoma xenografts.

NKT cell number is decreased in BM of patients with MYCN-amplified neuroblastoma metastatic to the BM. In order to test the clinical relevance of the potential of MYCN to repress CCL2 expression in neuroblastoma and prevent NKT cell localization to the metastatic site in BM, we examined T, NKT, and NK cell frequency in BM specimens from 15 patients with neuroblastoma (6 MYCN-amplified and 9 nonamplified) that had at least $10 \%$ tumor cells in BM by immunocytology testing. Specimens from 10 patients with stage 4 neuroblastoma without detectable BM metastases (4 MYCNamplified and 6 nonamplified) served as control. We used the same 5-color flow cytometric analysis that was applied for the mouse model described in Figure 5A. The data demonstrate that NKT cell frequency in BM of nonamplified neuroblastoma was $0.41 \% \pm 0.3 \%$, nearly 4-fold that of $M Y C N$-amplified $(0.12 \% \pm 0.06 \% ; P<0.05)$ or control specimens $(0.11 \% \pm 0.12 \% ; P<0.01$; Figure $7 \mathrm{~A})$. In contrast, the difference in NKT frequency between patients with $M Y C N$ amplified BM metastatic neuroblastoma and control patients was not significant $(P=0.3)$. The majority $(80 \% \pm 4.3 \%)$ of $\mathrm{BM}$-infiltrating NKT cells were CD4 ${ }^{+}$, which is consistent with the prevalence of this subset early in life (33). The frequency of NKT cells in the control group (tumor-free BM) did not differ between $M Y C N$-amplified and nonamplified specimens (data not shown). In contrast to NKT cells, the frequency of conventional T cells (Figure 7B) and monocytes (data not shown) did not differ among all 3 groups of patients. NK cells were very rare (less than $0.2 \%$ ) and also did not differ among the groups (data not shown). Although we could not obtain matching peripheral blood specimens from the same patients to exclude the possibility of a general increase of NKT cell number in nonamplified neuroblastomas, we have previously demonstrated that NKT cell frequency in patients with neuroblastoma, regardless of MYCN status, was equal to that in healthy volunteers (9). These data are consistent with our experimental findings indicating that MYCN represses CCL2 expression in neuroblastoma cells and prevents the recruitment of NKT cells to the site of disease, thus contributing to a microenvironment favorable to disease progression.

\section{Discussion}

This study reveals a cause-effect relationship between overexpression of a prognostically relevant oncogene, MYCN, in tumor cells and the failure of a selective subset of innate lymphocytes, NKT cells, to localize to the site of disease both in experimental animal models and in patients with metastatic neuroblastoma. Using gain-of-function and loss-of-function approaches, we demonstrated, for the first time to our knowledge, that MYCN negatively regulated the promoter activity of CCL2, a key chemokine that attracts NKT and effector-memory T cells $(9,15,18)$. Unlike previously described oncogene-driven immune escape pathways (26, 27), MYCN action in neuroblastoma cells neither activated STAT3 signaling nor caused suppression of DC maturation. Therefore, MYCN-mediated tumorigenesis is coupled with what we believe to be a novel mode of the immune escape.

The results of this study suggest that MYCN oncogene expression may provide an advantage to the tumor by avoiding NKT cells. Although NKT cells are not directly cytotoxic against CD1d- neuroblastoma cells (34), mounting evidence from the experimental models shows that NKT cells may provide an initial source of pro- 
A
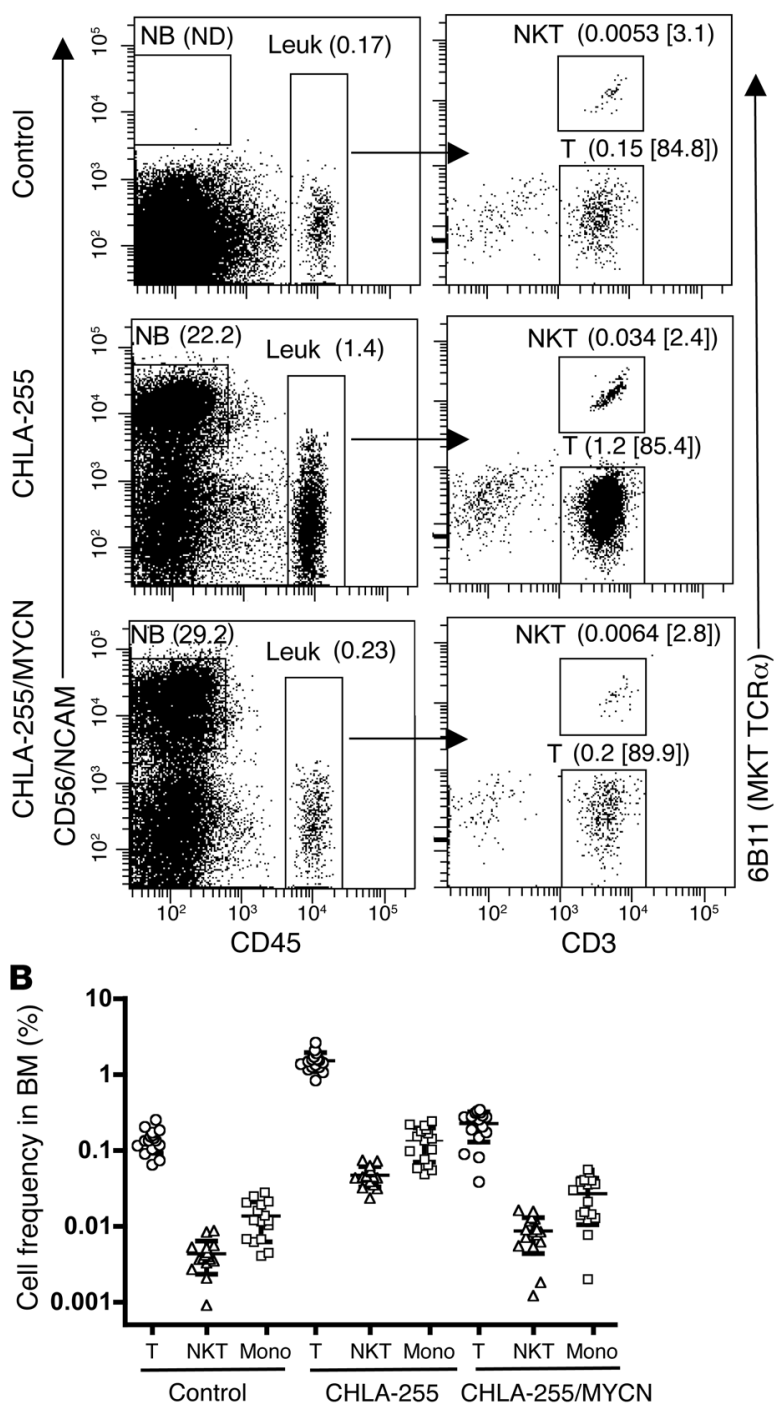

Figure 5

MYCN regulates NKT cell localization to neuroblastoma xenografts in BM of NOD/SCID mice. (A) CHLA-255 and CHLA-255/MYCN neuroblastoma cells were injected into the bone cavities of left and right femurs in NOD/SCID mice, and 4 weeks later in vitro-expanded human NKT cells were injected i.v. together with human PBMCs (1:10 ratio, $5 \times 10^{7}$ total cells). Mice were sacrificed after 24 hours, and BM from the indicated sources was analyzed by 5 -color flow cytometry. After exclusion of DAPI+ dead cells, the neuroblastoma (NB) cells and leukocytes (Leuk) were identified as CD56+CD45- and CD45 ${ }^{+}$events, respectively. T cells $\left(\mathrm{CD}^{+}+6 \mathrm{~B} 11^{-}\right)$and NKT cells $\left(\mathrm{CD}^{+}+6 \mathrm{~B} 11^{+}\right)$were analyzed after gating on $\mathrm{CD} 45^{+}$region. $\mathrm{BM}$ of tumor-free tibias served as control. Numbers within parentheses and brackets represent percentages of all viable cells (DAPI-) and of leukocytes, respectively. Data are from a representative individual mouse. ND, not detected. (B) Scatter plot of T, NKT cell, and monocyte (Mono) frequency among all non-neuroblastoma cells in BM from individual mice as indicated in 3 different experiments. Data are mean \pm SEM from 16 animals.

density of CD45RO ${ }^{+}$cells (44). The selective migration of NKT and $\mathrm{CD} 45 \mathrm{RA}^{-} \mathrm{CCR}^{-} \mathrm{T}$ cells in our mouse model are consistent with previous reports that NKT cells have a trafficking pattern very similar to that of effector-memory T cells $(17,18)$. Although Pages et al. did not examine NKT cells among tumor-infiltrating CD45RO ${ }^{+} \mathrm{T}$ cells (44), another report demonstrated that NKT cell infiltration in primary colorectal tumors represents an independent factor of the favorable outcome (10). Therefore, by preventing NKT cell localization to the tumor site, as we showed in patients with neuroblastoma, or by affecting their functional properties, as has been shown in patients with myeloma (7), tumor may disrupt a natural bridge between innate inflammation and adaptive immune responses that may be essential for tumor progression.

We demonstrated that, in contrast to other immunosuppressive oncogenes $(26,27)$, MYCN targeted the ability of tumor cells to chemoattract NKT cells without inducing factors that would directly affect lymphocyte viability or DC function. However, we cannot exclude the possibility that MYCN could indirectly mediate killing of tumor-infiltrating T or NKT cells in vivo. Because murine microenvironment may not be appropriate to study indirect effects mediated by human cells, we are now in the process of adapting a humanized mouse model engrafted with human $\mathrm{CD} 34^{+}$hematopoietic precursors to investigate this issue.

The importance of NKT cell trafficking to the site of disease has been demonstrated in various pathological conditions. For example, NKT cells that were recruited by $\mathrm{Ccl} 2$ to the lungs mediated a delayed-type hypersensitivity response in a model of pulmonary infection with Cryptococcus neoformans (45). Selective genetic deletion of NKT cells in J $18^{-/-}$mice suppressed Th1 responses and prevented clearance of the infection. $\mathrm{Ccl} 2$ production preceded NKT cell-mediated inflammatory responses in wild-type mice, whereas the lack of lung infiltration with NKT cells in $\mathrm{Ccl}^{-/-}$mice, although NKT cells remained functional, was associated with a failure to develop protective immunity against the fungus (46). Our data strongly suggest that by repressing CCL2, a key chemokine responsible for NKT cell chemoattraction, MYCN may effectively prevent NKT cell function in neuroblastoma. It is still likely that other factors contribute to NKT cell localization to the tumor. In our previous report, we performed gene expression analysis of 43 chemokine genes in primary neuroblastomas and found that 3 other chemokines (CCL5, CXCL12, and CCL21) weakly correlated with NKT cell infiltration (9). However, these chemokines were 


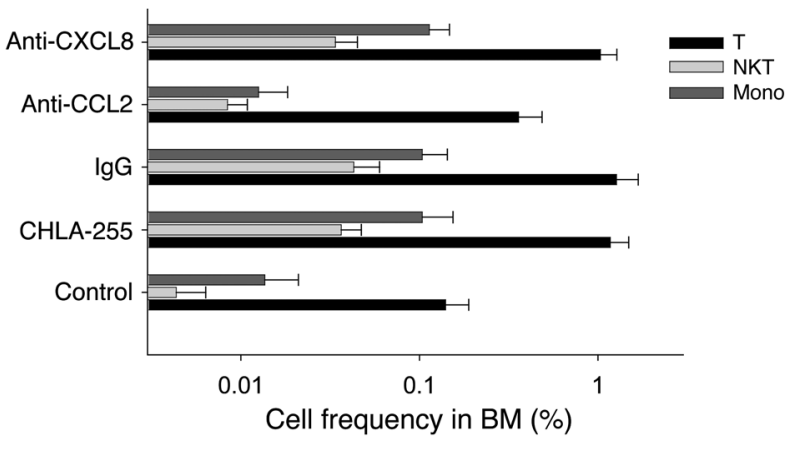

Figure 6

CCL2 is required for leukocyte migration to neuroblastoma xenografts in BM of NOD/SCID mice. Xenografts of CHLA-255 neuroblastoma cells were grown in femurs of NOD/SCID mice for 4 weeks. Neutralizing antibodies against CCL2, CXCL8, or isotype control (IgG) at 20 $\mu \mathrm{g}$ per mouse were injected i.p. 24 hours before adoptive transfer of human PBMCs, with subsequent analysis of leukocyte migration as in Figure 5. Data are mean \pm SD from 5 experiments. Each experiment included all groups, with 1 mouse per group and PBMCs from the same donor.

found at very low or undetectable levels in the cell lines, and corresponding blocking antibodies did not inhibit NKT or T cell migration. Therefore, to our knowledge there is currently no evidence for the role of other chemokines and/or cytokines in the direct tumor cell-mediated chemoattraction of NKT or T cells. We hypothesize that tumor stroma is the main source of these chemokines and other factors that may shape NKT trafficking in neuroblastoma and other types of cancer.

MYCN may play an etiologic role at least in a subset of aggressive neuroblastomas, because neuroectodermal-specific expression of this oncogene leads to the development of neuroblastoma-like disease in transgenic mice (47). This suggests that MYCN-driven active suppression of NKT cell localization to the tumor site could be as early and as essential a step in oncogenesis as the loss of the growth control. Moreover, accumulating evidence suggests that effective immune escape is a general requirement for oncogenemediated tumorigenesis and tumor progression. For example, an aberrant activation of a common STAT3-mediated oncogenic signaling pathway has been recognized in a variety of human cancers to cause negative regulation of inflammatory responses and suppression of the crosstalk between innate and adaptive immunity, thereby allowing tumors to escape from the immune system control $(26,27,48)$. A recent report has shown that PAX3-FKHR, an oncogenic fusion protein associated with the most aggressive (alveolar) type of rhabdomyosarcoma, interacts with STAT3 to cause a dramatic reduction of tumor MHC expression and suppression of IFN-inducible genes and chemokines (27). We found that STAT3 was not involved in MYCN-mediated CCL2 gene repression in neuroblastoma. On the other hand, STAT3 activation has been shown to upregulate MYCC in tumor cells (26), suggesting that MYC could be one of the downstream mediators in the STAT3-mediated immunosuppression. Thus, targeting MYCN and probably other MYC family oncogenes could be important not only for controlling tumor cell proliferation, but also for eliciting effective antitumor immune responses.

In addition to our previous demonstration of lack of NKT cell infiltration in primary $M Y C N$-amplified neuroblastomas (9), we now show that NKT cells also failed to localize to the BM containing $M Y C N$-amplified neuroblastoma cells. Taken together with the fact that NKT cells selectively accumulated in the metastatic BM of nonamplified patients, these data suggest that NKT cells may be involved in the control of the metastatic process in BM, which is the most common site of metastases and relapse in neuroblastoma and many other types of cancer (49). This view is supported by reports of potent antimetastatic activity of specific ligands for NKT cells such as $\alpha$-galactosylceramide ( $\alpha$ GalCer, KRN-7000) and $\mathrm{C}-\alpha \mathrm{GalCer}$ in multiple models of cancer $(3,50,51)$.

Despite the ability of CCL2-producing neuroblastoma cells to attract $\mathrm{T}$ cells and monocytes in our in vitro and in vivo models, we found no difference between $\mathrm{T}$ cell and monocyte numbers in $M Y C N$-amplified versus nonamplified BM metastases in patients. This could be explained by the fact that $\mathrm{T}$ cells are a much more heterogeneous population compared with the NKT subset, so that the effect of tumor-derived chemokines on $\mathrm{T}$ cell frequency in BM could be nullified by other chemokines derived from normal BM cells, such as CXCL12, which is expressed at high levels by sinusoidal endothelium in the BM (52). In addition, it is well documented that homeostatic expansion critically contributes to the number of BM T lymphocytes. In fact, the rate of homeostatic proliferation of T cells in human BM is second only to that in the thymus (53). Monocytes originate in BM, and their migration back from periphery may not be sufficient to significantly affect their frequency. Little is known about the homeostasis of NKT cells in human BM. Unlike in the mouse, NKT cells are rare in human BM (54), suggesting that additional homing of tumorattracted NKT cells could substantially contribute to the overall number of these cells when metastatic tumor cells have the chemoattractive properties. However, a systematic evaluation of NKT cell homeostasis in human BM in physiological and pathological conditions is yet to be undertaken.

Despite the fact that NKT localization to the tumor site correlates with favorable outcome in patients with neuroblastoma (9) and colon cancer (10) and, conversely, that NKT numbers and functional activities are selectively decreased in many types of cancer $(6,55)$, there is still no direct evidence that NKT cells play a positive role in tumor immunity in humans. Considering that these cells can produce both Th1 and Th2 cytokines and have previously been shown to suppress immune responses in several models of autoimmune diseases (56), care should be taken in planning NKT cell-based immunotherapies of cancer. However,
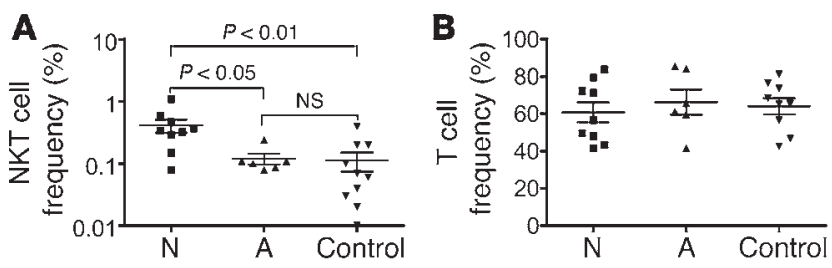

\section{Figure 7}

NKT cell number is decreased in BM of $M Y C N$-amplified neuroblastomas with BM metastases. BM specimens were analyzed by 5 -color flow cytometry as in Figure 5. Shown are scatter plots of (A) NKT cell and $(\mathrm{B}) \mathrm{T}$ cell frequency distribution in BM from $\mathrm{MYCN}$-amplified (A) versus nonamplified $(\mathrm{N})$ neuroblastomas with more than $10 \%$ tumor cells in BM. Tumor-free BM specimens from age-matched patients with stage 4 (amplified and nonamplified) neuroblastomas were used as control. Data are mean \pm SEM from BM specimens of 25 patients. 
recent experimental evidence suggests that previously reported tumor-protective properties of CD1d-reactive T cells $(57,58)$ do not depend on classical $\alpha$ GalCer-reactive NKT cells (59), which are the subject of the present article. Moreover, recent clinical trials with $\alpha$ GalCer-pulsed DCs in cancer patients demonstrated sustained in vivo expansion of NKT cells and activation of other cells of the immune system in a Th1-like manner $(60,61)$. Our present results suggest that in addition to NKT cell activation and expansion, a strategy should be developed to target NKT cell localization to the tumor site.

\section{Methods}

Cell lines, cell culture, and in vitro migration experiments. LA-N-1, CHLA-255, and SK-N-BE(2) neuroblastoma cell lines were established and maintained as described previously $(30,62-64)$. In vitro migration experiments were performed as described previously (9). Briefly, supernatants were collected from 24 -hour culture of $70 \%-80 \%$ confluent neuroblastoma cells and added $(0.6 \mathrm{ml})$ to the bottom chambers of 24-well plates with polycarbonate Transwell filters with $5-\mu \mathrm{m}$ pore size (Costar Corning). Where indicated, supernatants were preincubated for 1 hour with $10 \mu \mathrm{g} / \mathrm{ml}$ of IgG1 isotype control, clone 11711 , or neutralizing $\mathrm{mAb}$ against CCL2, 24822, or against CXCL8, 6217 (R\&D Systems). PBLs were obtained from freshly isolated PBMCs by collecting nonadherent cells after 45 minutes' incubation in 10-cm petri dishes (Costar Corning). For these experiments we only used donors who had at least $0.1 \%$ NKT cell frequency in PBMCs as determined by FACS (see below). PBLs from the same donor were used for all conditions in each experiment. PBLs were added to the upper chamber $\left(10^{6} \mathrm{cells}\right.$ in $100 \mu \mathrm{l}$ ), and the Transwell chambers were incubated at $37^{\circ} \mathrm{C}$ in $5 \% \mathrm{CO}_{2}$. After 4 hours, initial input cells (from a nonpermeable well) and migrated cells from the lower chambers were stained with fluorochrome-conjugated mAbs (see Flow cytometry). NKT cells were identified using FITC-conjugated anti-CD3 UCHT1 and PE-conjugated anti-Va24-Ja18 6B11 mAbs (BD Biosciences). Monocyte migration from freshly isolated PBMCs was evaluated in separate experiments. Monocytes were stained with FITC-conjugated anti-CD14 M5E2 mAb (BD Biosciences). After suspension in TruCOUNT tubes with an exact number of beads (BD Biosciences), multicolor immunofluorescence was analyzed by flow cytometry to determine relative and absolute numbers of cells in subpopulations of interest. For example, the absolute number of NKT cells per tube is calculated as percent NKT cells multiplied by 48,449 (known number of beads) and divided by percent beads. The percent migration for each subset was calculated by dividing the number of migrated cells by the number of input cells. Specific migration was calculated by subtracting migration to control medium from migration to the experimental medium.

Plasmids, cloning, shRNA, and lentiviral transduction of neuroblastoma cells. MYCN-estrogen receptor (MYCN-ER) ligand-binding domain cDNA was a gift from W. Weiss (UCSF, San Francisco, California, USA). MYCN-ER was subcloned into the BamH I site of PCCL-MNDU3-IRES-CD80 to generate the construct PCCL-MNDU3-MYCN/ER-IRES-CD80. The lentiviral vector was produced by the vector core facility of CHLA.

Neuroblastoma cells were seeded into 6-well plates at a concentration of $2.0 \times 10^{5}$ cells/well 1 day before transduction. Virus supernatant from PCCL-MNDU3-MYCN/ER-IRES-CD80 or vector PCCL-MNDU3-IRES$\mathrm{CD} 80$ was added into each well at $10^{7}$ infective units $/ \mathrm{ml}$. After 24 hours' incubation at $37^{\circ} \mathrm{C}$ in $5 \% \mathrm{CO}_{2}$, cells were stained with PE-conjugated antiCD80 L307.4 (BD Biosciences) and positively sorted by FACS using a FACSAria cell sorter (BD Biosciences). After 3 rounds of cell expansion and sorting, the positive cells stably persisted at $99 \%$ purity. MYCN expression was tested by RT-PCR and Western blot of nuclear extract (see below). As with a previously reported MYCC-ER system (65), the presence of the ligand- binding domain of the ER in the MYCN-ER construct was designed to regulate functional activity of $\mathrm{MYCN}$ protein via enhanced nuclear translocation of MYCN in the presence of 4-OHT. However, likely because of high potency of MNDU3 promoter that resulted in a high level of MYCN protein expression, even in the absence of 4-OHT, MYCN was active enough to exert a maximum effect on the genes of interest. Therefore, we studied MYCN overexpression in an unregulated fashion, and this approach was complemented by MYCNkd experiments with MYCN shRNA.

The MYCN shRNA was designed to target MYCN mRNA (ATTGAACACGCTCGGACTTG). Primers were as follows: U6 upstream, 5'-TTTGGGGATCCAAGGTCGGGCAGGAAGAGG-3'; downstream of MYCN shRNA, 5'-GCGGATCCAAAAAAGATTGAACACGCTCGGACTTGCTACACAAACAAGTCCGAGCGTGTTCAATCGGTGTTTCGTCCTTTCCACAAGATA-3'. The U6 MYCN shRNA was inserted into pHIV-7-EGFP (a gift from J. Rossi, City of Hope, Los Angeles, California, USA) (66).

The transduction of neuroblastoma cells with lentiviral shRNA was performed as described above for MYCN cDNA. However, after MYCN downregulation, neuroblastoma cells grew at a much slower rate; to prevent outgrowth of nontransduced cells, we sorted EGFP-positive cells into 96-well plates at 1 cell per well using the FACSAria cell sorter (BD Biosciences). Those clones that after expansion remained 100\% EGFP-positive were screened for MYCNkd by RT-PCR and Western blot (see below).

Western blot. Neuroblastoma cell lines were harvested at $75 \%$ confluence, and nuclear proteins were isolated with nuclear extract kit (Active Motif). Protein concentration was determined using BCA Protein Assay kit (Pierce Biotechnology). Protein $(50 \mu \mathrm{g})$ was separated by SDS-PAGE in a $4 \%-20 \%$ Tris-Glycin gel according to standard protocols (Invitrogen) and transferred onto Immune-Blot PVDF membrane (Bio-Rad). MYCN expression pattern was analyzed using the purified mouse anti-human MYCN mAb (obtained from M. Schwab, Deutsches Krebsforschungszentrum, Heidelberg, Germany) at 1:1,000 dilution and goat anti-mouse horseradish peroxidase secondary antibody (Santa Cruz Biotechnology Inc.) at 1:2,000 dilution, followed by detection by ECL Western blotting as described by the manufacturer (Amersham Biosciences). As a control, each blot was stripped and reprobed with an actin-specific antibody (Santa Cruz Biotechnology Inc.).

To detect phosphorylated STAT3, neuroblastoma cells were lysed in radioimmunoprecipitation assay (RIPA) buffer $(20 \mathrm{mmol} / 1$ sodium phosphate buffer, $150 \mathrm{mmol} / \mathrm{l}$ sodium chloride, $5 \mathrm{mmol} / 1$ EDTA, $10 \%$ glycerol, $1 \%$ Triton $\mathrm{X}-100$, and $0.5 \%$ sodium deoxycholate) supplemented with 1 tablet of complete mini-EDTA protease inhibitor cocktail (Roche Diagnostics). An equal amount of protein was loaded in each well, electrophoretically separated in $4 \%-12 \%$ Bis-Tris gradient SDS page gel, and then transferred onto a nitrocellulose membrane by semidry transfer method. The membrane was blocked with Tris-buffered saline/Tween (TBS-T; $0.1 \%$ ) with $5 \%$ nonfat dried milk for 2 hours at room temperature with gentle shaking, followed by overnight incubation at $4{ }^{\circ} \mathrm{C}$ with anti-phosphorylated Stat 3 antibody at 1:1,000 dilution (Cell Signaling Technology). After washing with $0.1 \%$ TBS-T $3 \times$ and incubating with goat anti-rabbit horseradish peroxidase-conjugated secondary antibody (Cell Signaling Technology) at 1:2,000 dilution for 1 hour, protein bands were detected by ECL Western blotting method according to the manufacturer's instructions (Amersham Biosciences). Equal loading of the samples was confirmed by performing Western blot with anti-total STAT3 antibody (Cell Signaling Technology). Where indicated, neuroblastoma cells were stimulated with $10 \mathrm{ng} / \mathrm{ml}$ recombinant human IL-6 (R\&D Systems) for 15 minutes followed by the STAT3 analysis.

Real-time PCR. The MYCN forward primer (5'-GAAGAAATCGACGTGGTCACTG-3') is complimentary to sequences within MYCN exon 2; the reverse MYCN primer ( $5^{\prime}$-GGTGAATGTGGTGACAGCCTT- $\left.3^{\prime}\right)$ is complimentary to sequences within exon 3. The MYCN probe (FAM- 
5'-AGAAGCGGCGTTCCTCCTCCAACA-3'-TAMTph) hybridizes within exon 2. The probe/primers set for CCL2 was described previously (9), and one for GAPDH (TaqMan GAPDH Control Reagent) was purchased from ABI. Probes and primers were designed using Primer Express (version 1.5; Applied Biosystems) and were synthesized by Integrated DNA Technologies. A quantitative comparative threshold cycle analysis was performed using the ABI PRISM 7700 Sequence Detection System and PE Biosystems software (SDS 2.2.2).

$C B A$ analysis. Chemokines were detected with the CBA chemokine kit (BD Biosciences) according to the manufacturer's protocol and as previously described (34).

Flow cytometry. To analyze the frequency of human leukocytes and their subsets as well as the frequency of human neuroblastoma cells in the BM of NOD/SCID mice (see In vivo experiments), we used 4 sets of multicolor flow cytometry. Set 1 consisted of PE-Cy5-conjugated anti-CD45 HI30, allophycocyanin-conjugated (APC-conjugated) anti-CD56 B159, FITCconjugated anti-CD3 UCHT1, and PE-conjugated 6B11 (Va24-Ja18) mAb (BD Biosciences), and DAPI (Invitrogen) was added 5 minutes before the flow analysis. Set 2 consisted of PE-Cy5-conjugated anti-CD45 HI30, FITC-conjugated anti-CD3 UCHT1, APC-conjugated anti-CD14 M5E2, PE-conjugated anti-CD19 SJ25C1, and DAPI. Set 3 consisted of PECy5-conjugated anti-CD45 HI30, FITC-conjugated anti-CD3 UCHT1, PE-conjugated 6B11, pacific blue-conjugated anti-CD4 PRA-T4, and APC-Cy7-conjugated anti-CD8 SK1 (BD Biosciences). Set 4 consisted of PE-Cy5-conjugated anti-CD45 HI30, FITC-conjugated anti-CD3 UCHT1, APC-conjugated anti-CD45RA HI100 (BD Biosciences), PE-conjugated anti-CCR7 150503 (R\&D Systems), and DAPI. Reactivity to mouse and human $\mathrm{F}_{\mathrm{c}}$ receptors was blocked by 5 minutes' preincubation with mouse Fc-block (1:1,000; BD Biosciences) and polyclonal human IgG $(2 \mathrm{mg} / \mathrm{ml}$; Sigma-Aldrich), respectively. Fluorochrome- and isotype-matching mAbs as suggested by the manufacturer $(\mathrm{BD})$ were used as negative controls. In the experiments with human PBMCs and BM, unstained cells and special staining controls that employ all reagents except for the one of interest (fluorescence minus one) were used as described previously (67). At the time of analysis, cells were thawed and placed for 1 hour in complete RPMI 1640 plus DNAse-I (0.17 U/ml; Invitrogen). From 200,000 to 1,000,000 DAPI- events per sample were collected. Induction of apoptosis was evaluated in primary $\mathrm{T}$ and NKT cells after 24 hours' coculture of PBMCs with neuroblastoma cells with neuroblastoma cell/PBMC ratios of 1:5 and 1:1. Culture of PBMCs in the medium alone was used as a control. Cells were stained with PerCP-Cy5.5-conjugated anti-CD3 UCHT1, PE-conjugated $6 \mathrm{~B} 11$, and FITC-conjugated annexin V (BD Biosciences) according to the manufacturer's instructions. The analysis was performed on a LSR-II 4-laser flow cytometer (BD Biosciences) using FACDiva software (version 5.0.1; BD). Flow cytometry was carried out in the USC-CHLA Institute for Pediatric Clinical Research and Saban Research Institute Analytical Cytology Core Laboratory.

DC maturation assay. DCs were generated from PBMCs as described previously (61), with minor modifications. Briefly, $5 \times 10^{7}$ PBMCs were cultured in $10-\mathrm{cm}$ petri dishes (BD Falcon; BD Biosciences) in RPMI 1640 containing $5 \%$ human serum (BioWhittaker) for 45 minutes. After removing floating cells and washing twice with PBS, adherent cells were cultured with 800 $\mathrm{U} / \mathrm{ml} \mathrm{GM}-\mathrm{CSF}$ and 1,000 U/mL IL-4 (Peprotech). Where indicated, half of the culture medium was replaced with tumor cell supernatants or Iscove's modified Dulbecco's medium as a control. At day 5, the cell phenotype was analyzed by 3 -color flow cytometry using FITC-conjugated anti-CD86 2331 and PE-conjugated anti-CD80 L307.4 mAbs (BD Biosciences) and DAPI for dead cell exclusion (Invitrogen).

Promoter assay. $\mathrm{P}(3.5)$-CCL2 promoter construct was kindly provided by R. Ransohoff (Lerner Research Institute, Cleveland, Ohio, USA). The
E-box-containing 2.8-kb and E-box-deleted 2.6-kb regions were amplified by PCR using the Expand long template PCR system (Roche Applied Sciences) on $10 \mathrm{ng}$ human genomic DNA (BD Biosciences). The primers used were based on the GenBank sequence (accession no. AY357296). For the 2.8-kb promoter region, the forward primer was 5'-CGGGGTACCGAGATGTTCCCAGCACAG with an added KpnI site, and the reverse primer (position +46 to +66) was 5'-CGGCGCCCCGGGTTTCATGCTGGAGGCGAGAGT with an added Smali site. For the $2.6-\mathrm{kb}$ region, the forward primer was 5'-CGGACGCGTATTCTCTTCTACGGGATCTGG with Mlui site and the reverse one was the same as for $2.8-\mathrm{kb}$ region. The amplified products were cloned into pGL3-Basic to obtain pCCL2-luc construct. Clones were verified by sequencing. Neuroblastoma cells were cotransfected with the pCCL2-luc and pRL-SV40 plasmids using Lipofectamine 2000 (Invitrogen). The activity of both Firefly and Renilla luciferase was determined 48 hours later using the Dual Luciferase Assay kit (Promega). A minimum of 3 independent transfections was performed in duplicate, and specific CCL2 promoter activity was normalized to Renilla luciferase.

EMSA. To perform EMSA experiments, we used the LightShift Chemiluminescent EMSA kit (Pierce Biotechnology). Nuclear extracts from neuroblastoma cells were used to detect E-box binding according to the manufacturer's instructions. The E-box oligonucleotide probe corresponds to the E-box site of the human CCL2 promoter: TTCCCAGCACAGCCCCATGTGAGAGCTCCCTGGCTC. INR probes were as follows: INR-1, CTTTCTTTTCTGATTCATACCCTTCACCTTCCCTGTGTTTACTGTCTGATATATG; INR-2, GGTCAGTCTGGGCTTAATGGCACCCCATCCTCCCCATTTGCTCATTTGGTCTCAG; INR-3, CTCCTCAGAGTGGGAATTTCCACTCACTTCTCTCACGCCAGCACTGACCTCCCAG.

In vivo experiments. Four-week-old female NOD/SCID mice were purchased from The Jackson Laboratory. CHLA-255 and CHLA-255/MYCN neuroblastoma cells were injected into the bone cavities of left and right femurs of the same NOD/SCID mice as described previously (30). Tumor growth in $\mathrm{BM}$ was indirectly assessed via detection of progressive bone lesions by weekly X-ray imaging at the small animal imaging core facility of CHLA. Mice with grade 4 lesions (bone fracture) were excluded from the analysis. After 4 weeks, when most animals had grade 3 lesions, in vitroexpanded human NKT cells (34) were injected i.v. together with freshly isolated PBMCs (1:10 ratio, $5 \times 10^{7}$ total cells). Before injection to the animals, NKT cells had been cultured with IL-2, $100 \mathrm{U} / \mathrm{ml}$ (Peprotech) for 7-10 days without TCR stimulation to achieve resting phase, when their trafficking pattern more closely resembled that of primary NKT cells, as we determined in the earlier study (9). One day prior to human leukocyte transfer, mice were injected i.p. with anti-IL-2R $\beta$ TM- $\beta 1 \mathrm{mAb}$ at $0.2 \mathrm{mg} /$ mouse to deplete residual mouse NK cells (68) (TM- $\beta 1$ hybridoma was a gift of T. Tanaka, Osaka University Medical Center, Osaka, Japan). Where indicated, TM- $\beta 1 \mathrm{mAb}$ was injected together with $20 \mu \mathrm{g}$ of blocking antihCCL2, anti-CXCL8, or mouse IgG1 isotype control, the same mAbs used for in vitro blocking experiments. In vivo blocking experiments were performed using PBMCs from the same donor for all groups in an experiment. Frozen PBMCs were purchased for these experiments from Astarte Biologics. Mice were sacrificed after 24 hours, and BM from the indicated sources was analyzed by set- 1 and -2 of multicolor flow cytometry as described in Flow cytometry. Animal experiments were approved by the Institutional Animal Care and Use Committee of CHLA.

Human specimens. We analyzed 25 cryopreserved BM specimens from newly diagnosed patients with stage 4 neuroblastoma who were enrolled in Children's Cancer Group studies for neuroblastoma (studies CCG3891, CCG-321P1, CCG-321P2, CCG-321P3, and CCG-323). Fifteen patients had BM metastases with at least $10 \%$ tumor cells in $\mathrm{BM}$ detected by immunocytology; 6 of the primary tumors from these same patients 
had MYCN amplification and 9 did not. Ten patients with stage 4 neuroblastoma without BM metastases served as a control group. Tumors were analyzed for MYCN genomic amplification by Southern blot, PCR, and/or immunohistochemistry as described previously (69). PBMCs were isolated from freshly obtained blood from healthy adult donors as described previously (34). Informed consent was obtained in accordance with institutional review board policies and procedures for research dealing with human specimens.

Statistics. Data obtained in the in vitro experiments were analyzed with 1-way ANOVA with the Tukey-Kramer post-test comparison of group means.

For xenograft data, cell frequencies for CHLA-255, CHLA-255/MYCN, and control sites were obtained from each mouse. The percents of leukocytes and subtypes were normalized before analysis by taking their ratios with the percents of non-NB cells to remove the influence of the presence or absence of NB cells in the marrow independent of any effect of these cells on leukocyte infiltration. The ratios were also transformed to the $\log _{10}$ scale before statistical analysis to correct skewness. The primary test of differences in leukocyte migration in CHLA-255 and CHLA-255/MYCN sites was of the null hypothesis that the mean difference in $\log _{10}$ ratios in the CHLA-255 (or CHLA-255/MYCN) site and the control site is 0. Statistical differences were determined using paired, 2-tailed Student's $t$ test.

Data obtained from neuroblastoma patients were analyzed with 1-way ANOVA with the Tukey-Kramer post-test comparison of group means, comparing mean NKT and T cell percentages between patients without $\mathrm{BM}$ infiltration (control) and those with infiltration with $M Y C N$-amplified or nonamplified tumor.
Statistical computations were performed with Prism (version 4.0; GraphPad Software) and Stata (version 9.0; StataCorp).

\section{Acknowledgments}

We thank J. Barnhart (CHLA) for FACS sorting, P. Wakamatsu (CHLA) for assistance with databases, and R. Acevedo (BD Biosciences) for invaluable advice on digital cytometry and data analysis. This work was supported by NIH grants CA116548 (to L.S. Metelitsa) and CA81403 and CA60104 (to R.C. Seeger); by Concern Foundation; by the Saban Research Institute Carrier Development Award; by Wright Foundation (to L.S. Metelitsa); and by the Neil Bogart Memorial Fund of the T.J. Martell Foundation for Leukemia, Cancer, and AIDS Research (R.C. Seeger, L.S. Metelitsa, and Y.A. DeClerck).

Received for publication October 25, 2006, and accepted in revised form May 29, 2007.

Address correspondence to: Leonid S. Metelitsa, Division of Hematology-Oncology, Childrens Hospital Los Angeles, 4650 Sunset Boulevard, Los Angeles, California 90027, USA. Phone: (323) 3611839; Fax: (323) 361-9455; E-mail: lmetelitsa@chla.usc.edu.

Chan-Wook Woo's present address is: Department of Pediatrics, Ansan Hospital, and College of Medicine, Korea University, Ansan City, Republic of Korea.
1. Kronenberg, M. 2005. Toward an understanding of NKT cell biology: progress and paradoxes. Annu. Rev. Immunol. 23:877-900.

2. Van Kaer, L., and Joyce, S. 2005. Innate immunity: NKT cells in the spotlight. Curr. Biol. 15:R429-R431.

3. Swann, J., Crowe, N.Y., Hayakawa, Y., Godfrey, D.I., and Smyth, M.J. 2004. Regulation of antitumour immunity by CD1d-restricted NKT cells. Immunol. Cell Biol. 82:323-331.

4. Gumperz, J.E. 2004. CD1d-restricted "NKT” cells and myeloid IL-12 production: an immunological crossroads leading to promotion or suppression of effective anti-tumor immune responses? J. Leukoc. Biol. 76:307-313.

5. Yanagisawa, K., et al. 2002. Impaired proliferative response of $\mathrm{V}$ alpha $24 \mathrm{NKT}$ cells from cancer patients against alpha-galactosylceramide. J. Immunol. 168:6494-6499.

6. Tahir, S.M., et al. 2001. Loss of IFN-gamma production by invariant NK T cells in advanced cancer. J. Immunol. 167:4046-4050.

7. Dhodapkar, M.V., et al. 2003. A reversible defect in natural killer $t$ cell function characterizes the progression of premalignant to malignant multiple myeloma. J. Exp. Med. 197:1667-1676.

8. Molling, J.W., et al. 2007. Low levels of circulating invariant natural killer $\mathrm{T}$ cells predict poor clinical outcome in patients with head and neck squamous cell carcinoma. J. Clin. Oncol. 25:862-868.

9. Metelitsa, L.S., et al. 2004. Natural killer T cells infiltrate neuroblastomas expressing the chemokine CCL2. J. Exp. Med. 199:1213-1221.

10. Tachibana, T., et al. 2005. Increased intratumor Valpha24-positive natural killer T cells: a prognostic factor for primary colorectal carcinomas. Clin. Cancer Res. 11:7322-7327.

11. Ghiringhelli, F., Apetoh, L., Housseau, F., Kroemer, G., and Zitvogel, L. 2007. Links between innate and cognate tumor immunity. Curr. Opin. Immunol. 19:224-231.

12. Yu, H., Kortylewski, M., and Pardoll, D. 2007. Crosstalk between cancer and immune cells: role of STAT3 in the tumour microenvironment. Nat.
Rev. Immunol. 7:41-51.

13. Matsushima, K., Larsen, C.G., DuBois, G.C., and Oppenheim, J.J. 1989. Purification and characterization of a novel monocyte chemotactic and activating factor produced by a human myelomonocytic cell line. J. Exp. Med. 169:1485-1490.

14. Rollins, B.J., Walz, A., and Baggiolini, M. 1991. Recombinant human MCP-1/JE induces chemotaxis, calcium flux, and the respiratory burst in human monocytes. Blood. 78:1112-1116.

15. Carr, M.W., Roth, S.J., Luther, E., Rose, S.S., and Springer, T.A. 1994. Monocyte chemoattractant protein 1 acts as a T-lymphocyte chemoattractant. Proc. Natl. Acad. Sci. U. S. A. 91:3652-3656.

16. Roth, S.J., et al. 1998. Transendothelial chemotaxis of human alpha/beta and gamma/delta T lymphocytes to chemokines. Eur. J. Immunol. 28:104-113.

17. Kim, C.H., Butcher, E.C., and Johnston, B. 2002. Distinct subsets of human Valpha24-invariant NKT cells: cytokine responses and chemokine receptor expression. Trends Immunol. 23:516-519.

18. Kim, C.H., Johnston, B., and Butcher, E.C. 2002. Trafficking machinery of NKT cells: shared and differential chemokine receptor expression among Valpha24(+)Vbeta11(+) NKT cell subsets with distinct cytokine-producing capacity. Blood. 100:11-16.

19. Payne, G.S., et al. 1981. Analysis of avian leukosis virus DNA and RNA in bursal tumours: viral gene expression is not required for maintenance of the tumor state. Cell. 23:311-322.

20. Grandori, C., Cowley, S.M., James, L.P., and Eisenman, R.N. 2000. The Myc/Max/Mad network and the transcriptional control of cell behavior. Annu. Rev. Cell Dev. Biol. 16:653-699.

21. Nesbit, C.E., Tersak, J.M., and Prochownik, E.V. 1999. MYC oncogenes and human neoplastic disease. Oncogene. 18:3004-3016.

22. Seeger, R.C., et al. 1985. Association of multiple copies of the N-myc oncogene with rapid progression of neuroblastomas. N. Engl. J. Med. 313:1111-1116.

23. Blackwood, E.M., and Eisenman, R.N. 1991. Max: a helix-loop-helix zipper protein that forms a sequence-specific DNA-binding complex with Myc.
Science. 251:1211-1217.

24. Wanzel, M., Herold, S., and Eilers, M. 2003. Transcriptional repression by Myc. Trends Cell Biol. 13:146-150.

25. Metelitsa, L.S. 2004. Flow cytometry for natural killer T cells: multi-parameter methods for multifunctional cells. Clin. Immunol. 110:267-276.

26. Wang, T., et al. 2004. Regulation of the innate and adaptive immune responses by Stat- 3 signaling in tumor cells. Nat. Med. 10:48-54.

27. Nabarro, S., et al. 2005. Coordinated oncogenic transformation and inhibition of host immune responses by the PAX3-FKHR fusion oncoprotein. J. Exp. Med. 202:1399-1410.

28. Gery, S., and Koeffler, H.P. 2003. Repression of the TMEFF2 promoter by c-Myc. J. Mol. Biol. 328:977-983

29. Dang, C.V. 1999. c-Myc target genes involved in cell growth, apoptosis, and metabolism. Mol. Cell. Biol. 19:1-11.

30. Sohara, Y., et al. 2003. Lytic bone lesions in human neuroblastoma xenograft involve osteoclast recruitment and are inhibited by bisphosphonate. Cancer Res. 63:3026-3031.

31. Beider, K., et al. 2003. Involvement of CXCR4 and IL-2 in the homing and retention of human NK and NK T cells to the bone marrow and spleen of NOD/SCID mice. Blood. 102:1951-1958.

32. Lanzavecchia, A., and Sallusto, F. 2002. Progressive differentiation and selection of the fittest in the immune response. Nat. Rev. Immunol. 2:982-987.

33. Baev, D.V., et al. 2004. Distinct homeostatic requirements of CD4+ and CD4- subsets of Valpha24-invariant natural killer T cells in humans. Blood. 104:4150-4156.

34. Metelitsa, L.S., et al. 2001. Human NKT cells mediate antitumor cytotoxicity directly by recognizing target cell CD1d with bound ligand or indirectly by producing IL-2 to activate NK cells. J. Immunol. 167:3114-3122.

35. Smyth, M.J., et al. 2002. NKT cells - conductors of tumor immunity? Curr. Opin. Immunol. 14:165-171.

36. Fujii, S., Liu, K., Smith, C., Bonito, A.J., and Stein- 
man, R.M. 2004. The linkage of innate to adaptive immunity via maturing dendritic cells in vivo requires CD40 ligation in addition to antigen presentation and CD80/86 costimulation. J. Exp. Med. 199:1607-1618.

37. Coussens, L.M., and Werb, Z. 2002. Inflammation and cancer. Nature. 420:860-867.

38. Dunn, G.P., Old, L.J., and Schreiber, R.D. 2004. The immunobiology of cancer immunosurveillance and immunoediting. Immunity. 21:137-148.

39. Pollard, J.W. 2004. Tumour-educated macrophages promote tumour progression and metastasis. Nat. Rev. Cancer 4:71-78.

40. Smyth, M.J., Godfrey, D.I., and Trapani, J.A. 2001. A fresh look at tumor immunosurveillance and immunotherapy. Nat. Immunol. 2:293-299.

41. de Visser, K.E., Eichten, A., and Coussens, L.M. 2006. Paradoxical roles of the immune system during cancer development. Nat. Rev. Cancer. 6:24-37.

42. Hakansson, A., Gustafsson, B., Krysander, L., and Hakansson, L. 1996. Tumour-infiltrating lymphocytes in metastatic malignant melanoma and response to interferon alpha treatment. $\mathrm{Br}$. J. Cancer. 74:670-676.

43. Xu, Y., Kroft, S.H., McKenna, R.W., and Aquino, D.B. 2001. Prognostic significance of tumour-infiltrating $\mathrm{T}$ lymphocytes and $\mathrm{T}$-cell subsets in de novo diffuse large B-cell lymphoma: a multiparameter flow cytometry study. Br. J. Haematol. 112:945-949.

44. Pages, F., et al. 2005. Effector memory T cells, early metastasis, and survival in colorectal cancer. N. Engl. J. Med. 353:2654-2666.

45. Kawakami, K., et al. 2001. Monocyte chemoattractant protein-1-dependent increase of $\mathrm{V}$ alpha 14 NKT cells in lungs and their roles in Th1 response and host defense in cryptococcal infection. J. Immunol. 167:6525-6532.

46. Uezu, K., et al. 2004. Accumulation of gammadelta $\mathrm{T}$ cells in the lungs and their regulatory roles in Th1 response and host defense against pulmonary infection with Cryptococcus neoformans. J. Immunol. 172:7629-7634.

47. Weiss, W.A., Aldape, K., Mohapatra, G., Feuerstein,
B.G., and Bishop, J.M. 1997. Targeted expression of MYCN causes neuroblastoma in transgenic mice. EMBO J. 16:2985-2995.

48. Calo, V., et al. 2003. STAT proteins: from norma control of cellular events to tumorigenesis. J. Cell. Physiol. 197:157-168.

49. Papac, R.J. 1994. Bone marrow metastases. A review. Cancer 74:2403-2413.

50. Parekh, V.V., Wilson, M.T., and Van Kaer, L. 2005. iNKT-cell responses to glycolipids. Crit. Rev. Immunol. 25:183-213.

51. Schmieg, J., Yang, G., Franck, R.W., and Tsuji, M. 2003. Superior protection against malaria and melanoma metastases by a C-glycoside analogue of the natural killer T cell ligand alpha-Galactosylceramide. J. Exp. Med. 198:1631-1641.

52. Avigdor, A., et al. 2004. CD44 and hyaluronic acid cooperate with SDF-1 in the trafficking of human CD34+ stem/progenitor cells to bone marrow. Blood. 103:2981-2989.

53. Di Rosa, F., and Pabst, R. 2005. The bone marrow: a nest for migratory memory $\mathrm{T}$ cells. Trends Immunol. 26:360-366.

54. Exley, M.A., et al. 2001. A major fraction of human bone marrow lymphocytes are Th2-like CD1d-reactive $\mathrm{T}$ cells that can suppress mixed lymphocyte responses. J. Immunol. 167:5531-5534.

55. Giaccone, G., et al. 2002. A phase I study of the natural killer T-cell ligand alpha-galactosylceramide (KRN7000) in patients with solid tumors. Clin. Cancer Res. 8:3702-3709.

56. Godfrey, D.I., and Kronenberg, M. 2004. Going both ways: immune regulation via CD1d-dependent NKT cells. J. Clin. Invest. 114:1379-1388. doi:10.1172/JCI200423594.

57. Terabe, M., et al. 2000. NKT cell-mediated repression of tumor immunosurveillance by IL-13 and the IL-4R-STAT6 pathway. Nat. Immunol. 1:515-520.

58. Terabe, M., et al. 2003. Transforming growth factor-beta production and myeloid cells are an effector mechanism through which CD1d-restricted T cells block cytotoxic T lymphocyte-mediated tumor immunosurveillance: abrogation prevents tumor recurrence. J. Exp. Med. 198:1741-1752.

59. Terabe, M., et al. 2005. A nonclassical non-Valpha14Jalpha18 CD1d-restricted (type II) NKT cell is sufficient for down-regulation of tumor immunosurveillance. J. Exp. Med. 202:1627-1633.

60. Nieda, M., et al. 2004. Therapeutic activation of Valpha24+Vbeta11+ NKT cells in human subjects results in highly coordinated secondary activation of acquired and innate immunity. Blood. 103:383-389.

61. Chang, D.H., et al. 2005. Sustained expansion of NKT cells and antigen-specific T cells after injection of alpha-galactosyl-ceramide loaded mature dendritic cells in cancer patients. J. Exp. Med. 201:1503-1517

62. Seeger, R.C., et al. 1977. Morphology, growth, chromosomal pattern and fibrinolytic activity of two new human neuroblastoma cell lines. Cancer Res. 37:1364-1371.

63. Goto, H., et al. 2003. Transduction of green fluorescent protein increased oxidative stress and enhanced sensitivity to cytotoxic drugs in neuroblastoma cell lines. Mol. Cancer Ther. 2:911-917.

64. Reynolds, C.P., et al. 1988. Biological classification of cell lines derived from human extra-cranial neural tumors. Prog. Clin. Biol. Res. 271:291-306.

65. Eilers, M., Schirm, S., and Bishop, J.M. 1991. The MYC protein activates transcription of the alphaprothymosin gene. EMBO J. 10:133-141.

66. Li, M.J., and Rossi, J.J. 2005. Lentiviral vector delivery of recombinant small interfering RNA expression cassettes. Methods Enzymol. 392:218-226.

67. Roederer, M. 2001. Spectral compensation for flow cytometry: visualization artifacts, limitations, and caveats. Cytometry. 45:194-205.

68. Tournoy, K.G., Depraetere, S., Meuleman, P., Leroux-Roels, G., and Pauwels, R.A. 1998. Murine IL-2 receptor beta chain blockade improves human leukocyte engraftment in SCID mice. Eur. J. Immunol. 28:3221-3230.

69. Crabbe, D.C., Peters, J., and Seeger, R.C. 1992. Rapid detection of MYCN gene amplification in neuroblastomas using the polymerase chain reaction. Diagn. Mol. Pathol. 1:229-234. 